\title{
Decomposing serial learning: What is missing from the learning curve?
}

\author{
KELLY M. ADDIS and MICHAEL J. KAHANA \\ Brandeis University, Waltham, Massachusetts
}

\begin{abstract}
Our current understanding of serial learning relies on the form of the learning curve and on changes in the serial position curve over repeated study-test trials (Ward, 1937). The averaging of data that produces these functions obscures the detailed history of memory for individual items over the course of study-test trials. Extending Tulving's (1964) analysis of free recall learning, we present a new analysis of serial learning that tracks the acquisition and forgetting of item and order information at the level of individual items. Applying this analysis to two large data sets on serial list learning allows us to discern among hypotheses that are indistinguishable solely on the basis of the learning curve.
\end{abstract}

Ever since Ebbinghaus (1885/1913), students of memory have sought to understand human sequence learning. Whereas early work analyzed the acquisition of long lists of items through repeated study and test trials (see Harcum, 1975, for a review), more recent studies have favored the analysis of immediate recall of relatively short lists (Brown, Preece, \& Hulme, 2000; Burgess \& Hitch, 1999; Cowan, Saults, Elliott, \& Moreno, 2002; Farrell \& Lewandowsky, 2002; Henson, 1998; Knoedler, Hellwig, \& Neath, 1999; Li, Schweickert, \& Gandour, 2000). This shift in attention reflects a desire to move to a finer grain of analyses-from measures of overall recall and learning to the analysis of the distribution of errors across individual list positions. This article bridges these two approaches by bringing a more detailed approach to the analysis of serial learning. Through analyses of both item and order gains and losses over trials, we show that one can distinguish among hypotheses of serial learning that are indistinguishable with analyses of mean recall accuracy alone.

The effect of repetition on learning is most often measured by plotting a learning curve. Consider the free recall task, in which participants are asked to recall a justpresented list in any order. In this task, recall probability increases logarithmically with the number of study trials (Tulving, 1962). The logarithmic form of the learning curve could arise from different underlying processes. For example, participants might maintain the items they have already recalled, but pick up fewer and fewer new items over trials. Alternatively, participants might pick up new items at a constant rate, while forgetting a percentage of the items that they had previously recalled. This would lead to a higher absolute number of items being

The authors acknowledge support from the National Institutes of Health, Grant MH55687. Correspondence concerning this article should be addressed to M. J. Kahana, Volen National Center for Complex Systems, MS 013, Brandeis University, Waltham, MA 02454-9110 (e-mail: kahana@brandeis.edu). forgotten over trials, such that the net gain of recalled items still increases over trials. Looking solely at the learning curve, one cannot distinguish between these possibilities. But by examining what happens to individual items from trial to trial, one can test these hypotheses.

In a classic analysis of the learning curve in free recall, Tulving (1964) examined these trial-to-trial transitions of individual items. He reminded us of two important features of episodic list-learning experiments. First, in most studies, which use words as stimuli, participants are not learning items per se; rather, they are learning that these items, mini-events in the experiment, occurred in a particular spatiotemporal context defining the study list. Tulving (1964) also noted that if queried immediately following an item's appearance on the list, participants would not have difficulty recalling that item. Thus, the process of learning is inextricably tied to the process of forgetting (and vice versa; Krueger, 1929).

Tulving (1964) decomposed the learning curve into four mutually exclusive types of information. Consider the fate of a study item on a given learning trial. In one case, an item that was not recalled on the previous trial is recalled on the current trial. In a second case, an item was recalled on the previous trial but is not recalled on the current trial. In a third case, an item was recalled on both the previous trial and the current trial. In the final case, the item was not recalled on either the previous or the current trial. Tulving (1964) showed that the number of items gained is nearly constant across trials. The number of previously recalled items that are forgotten is also essentially constant over trials, except in very early trials in which there are few or no items that could have been forgotten. The number of items that are maintained from trial to trial increases logarithmically. Finally, the number of items that have not been recalled on the previous or the current trial decreases monotonically over trials. From these four types of information, one can now determine why the learning curve is logarithmic. The slight decrease in the number of items gained over trials, coupled with 
the constant rate of forgetting previously recalled items, gives a net gain of items that decreases over trials.

In serial recall, the learning curve is approximately linear over trials, at least at early stages of learning (see the left panel of Figure 1; see also Waugh, 1961, 1963). One can again propose many possible hypotheses about what is happening to cause the shape of the learning curve. Take, for instance, Lewandowsky and Murdock's (1989) chaining model. The closed-loop implementation uses the storage equation

$$
\mathbf{m}_{T}=\mathbf{m}_{T-1}+\sum_{j=1}^{L L}\left[(1-d) \gamma_{j} \mathbf{f}_{j}+(1-d) \omega_{j} \mathbf{f}_{j} * \mathbf{f}_{j-1}\right],
$$

where $T$ is the current trial, $d$ is the dot product between $\mathbf{m}$ and the item $\mathbf{f}_{j}$ or the association $\mathbf{f}_{j} * \mathbf{f}_{j-1}, L L$ is the number of list items, and $\omega_{j}$ and $\gamma_{j}$ are the weighting parameters for item and associative information, respectively, with $\gamma_{j}=1-\omega_{j}$.

Take the case in which $\lambda=0.5$ and $\omega_{0}=0.5$. On trial $T=1$,

$$
\mathbf{m}_{1}=0.5 \sum_{j=1}^{L L}\left[\mathbf{f}_{j}+\mathbf{f}_{j} * \mathbf{f}_{j-1}\right] .
$$

Neglecting the effects of output encoding, the memory vector on Trials 2 and 3 are given by $\mathbf{m}_{2}=1.5 \mathbf{m}_{1}$ and $\mathbf{m}_{3}=1.75 \mathbf{m}_{1}$. Because there is no decay mechanism, item and associative information become stronger over trials, with their strength approaching 1.0 as $T \rightarrow \infty$.

During recall, correlating item $\mathbf{f}_{j-1}$ with the memory vector $\mathbf{m}$ will retrieve an approximate representation of the target $\mathbf{f}_{j}^{\prime}$, according to the retrieval equation $\mathbf{f}_{j-1} \# \mathbf{m}=\mathbf{f}_{j}^{\prime}$. Successful recall will occur if the resemblance of the retrieved information with the target $\left(\mathbf{f}_{j}^{\prime} \cdot \mathbf{f}_{j}\right)$ is greater than a threshold and also greater than the resemblance of the retrieved information with all competing items $\mathbf{f}_{k}$.
Because this retrieval rule is based on the "best" match, items recalled on a previous trial will continue to be recalled. Thus, this implementation predicts that the learning curve results from adding new items in the correct order and not forgetting items already recalled. Other versions of the model that include forgetting processes would predict that learning proceeds by adding many new items on each trial while forgetting some items that have been previously recalled.

A simple positional model (e.g., Lee \& Estes, 1981) stores associations between items and positions, with no direct item-to-item connections. At item presentation, the relative order of the item is assumed to be perfect. At each subsequent time point, however, the item's position may be perturbed to a nearby position. This creates an uncertainty gradient, formalizing the idea that participants' ability to recall an item's exact input position decreases over time. An item may be lost if its position is perturbed in such a way that the resulting information is not a recognizable code for any position. At test, the positional code is sequentially activated, generating the item in that position at that time point according to the perturbation rule above. A similar perturbation process occurs at the trial level, which, over trials, serves to reinforce the list items. Thus, in early trials, many new items will be recalled, but the order of several of these items may have been perturbed. Over trials, however, items will be placed in their correct positions. To distinguish among these hypotheses, we will extend Tulving's (1964) analysis to serial recall, examining the gains and losses of both item and order information of individual items from trial to trial.

We will reanalyze data from two serial learning experiments, whose methods are summarized below. We first will present learning curves obtained using three different scoring methods. We then will explain our new

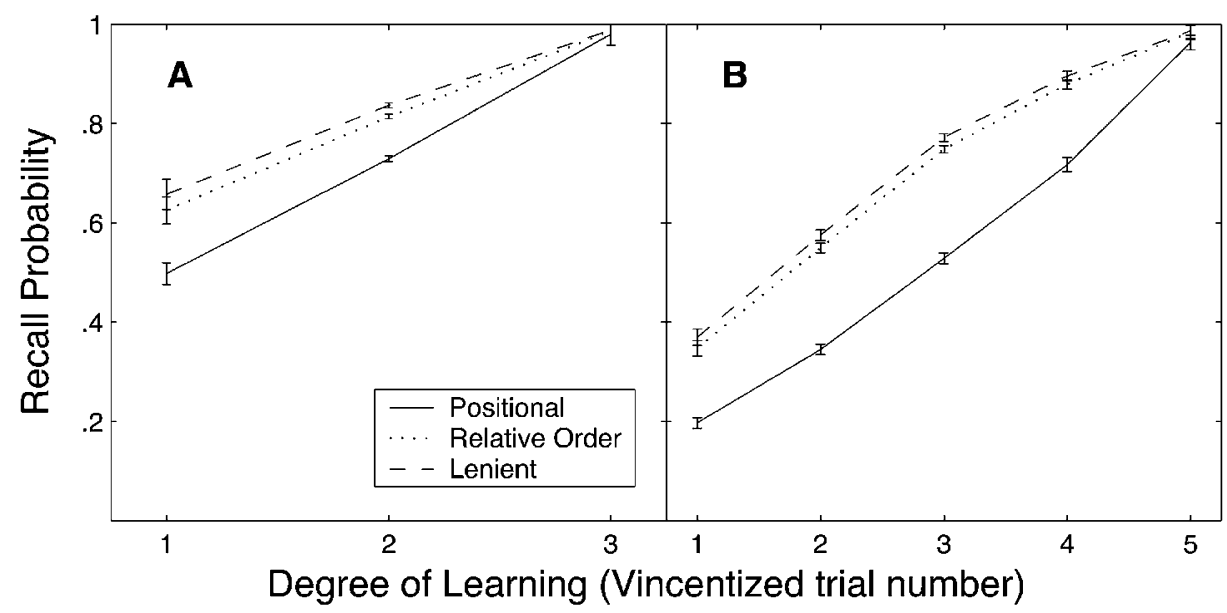

Figure 1. Learning curves. (A) Serial recall of consonants. (B) Serial recall of words. Error bars represent $95 \%$ confidence intervals calculated according to the methods of Loftus and Masson (1994). The trials of learning are Vincentized (see the text for an explanation). 
analysis of item and order gains and losses over trials. Finally, we will apply this analysis separately to easy and hard lists, and to good and poor learners.

In the first study (Kahana \& Jacobs, 2000), participants learned lists of 11,12 , and 13 consonants for an immediate serial recall test using the study-test method (lists were learned to a criterion of three consecutive perfect recalls, although only trials through the first perfect recall will be analyzed here). Consonants were displayed individually on the screen for $1 \mathrm{sec}$, followed by a 250 -msec blank interstimulus interval. At test, the participants typed their responses into a row of placeholders, indicating skipped positions with the space bar. Nineteen participants learned 432 lists across 12 sessions, yielding a total of 8,208 lists learned. Because this study was designed to examine the effects of repetition on serial recall, half of the lists contained repeated items. Those repetition lists will not be considered here. Only lists of length 13 with no repetitions are analyzed here; 6 lists from each session-a total of 72 lists per participantmet these criteria.

In the second study (Experiment 2 of Kahana \& Caplan, 2002), participants learned lists of 19 words for an immediate serial recall test using the study-test method (lists were learned to a criterion of one perfect recall). Words were presented auditorily at a rate of 1 word per $1.5 \mathrm{sec}$. At test, the participants vocally recalled as much of the list as they could, in the order of presentation. No instructions were given for indicating skipped positions. Sixty participants learned 24 lists across five sessions (the first 4 lists were considered practice and were excluded from analysis), yielding a total of 1,200 lists learned. Following mastery of each list, the participants were given a distractor task and a probed recall task. Here, we will analyze only the serial recall phase of the experiment.

\section{The Learning Curve}

In plotting the learning curves for recall of consonants (Kahana \& Jacobs, 2000) and recall of words (Kahana \& Caplan, 2002), we compared three scoring methods: strict positional scoring, relative order scoring, and lenient (item) scoring (Drewnowski \& Murdock, 1980). Strict positional scoring counts a recalled list item as correct only if it is in the correct serial position. Relative order scoring counts a recalled list item as correct if it is from a later serial position than the serial position of the previous recalled item. Lenient scoring counts a recalled list item as correct regardless of output position or order.

Because the lists in both experiments were learned to a criterion (one perfect recall for words, three perfect recalls for consonants), the number of trials to criterion varied from list to list and from participant to participant. To analyze data across these lists, we Vincentize the participants' performance (Vincent, 1912; Workman \& Adams, 1950). Vincentizing is a binning procedure in which segments of an individual's learning are substituted for actual trials. For example, a list learned in three trials could be Vincentized into six trials by weighting each real trial as two Vincentized trials. Alternatively, a list learned in nine trials could be Vincentized into six trials by weighting each real trial as two thirds of a Vincentized trial, so that two thirds of the first Vincentized trial would be the first real trial and one third would be from the second real trial. Thus, we would have the first sixth of learning, the second sixth of learning, and so on. We therefore use the term degree of learning, rather than trial number.

We binned the real data on the basis of the mean ${ }^{1}$ number of trials to criterion for each experiment. Furthermore, to facilitate comparison of these two studies, we included trials only through the first perfect recall, rather than through all three consecutive perfect recalls, in our analyses of Kahana and Jacobs (2000). For Kahana and Jacobs's study (recall of consonants), the mean number of trials to learn the list was three, whereas the mean number for Kahana and Caplan's (2002) study (recall of words) was five. The fact that analyses may show that less than $100 \%$ of the list was correctly recalled on the final Vincentized trial is an artifact of the binning procedure; the final real trial was $100 \%$ correct.

Figure 1 compares the three scoring methods across both experiments. Panel A shows the results from the first study, recall of consonants. All three curves are linear and increasing. ${ }^{2}$ Thus, on average, the participants had a net gain of items that remained constant over trials. The curves from relative order scoring and lenient scoring lie virtually on top of one another, whereas strict positional scoring produces lower percentages. This illustrates the fact that items are recalled in the correct relative order even when they are recalled in the incorrect serial position.

Figure 1B shows the results from the second study, recall of words. As in the first study, the relative order and the lenient scoring curves lie on top of one another, whereas strict positional scoring is slightly lower. In this case, however, the strict scoring accelerates slightly over trials, whereas the lenient and the relative order scoring decelerate over trials. This raises the possibility that late in learning, participants gain fewer new items but fit items previously recalled in incorrect positions into their correct positions. Solely on the basis of the learning curve, however, we cannot evaluate this hypothesis.

\section{Item/Order Analyses}

Just as the learning curve can be scored using different types of information (item, order, and position), so can trial-to-trial transitions of individual items. Extending Tulving's (1964) analysis to serial recall, we first will examine both item and relative order gains and losses over learning. Table 1 illustrates all of the possible permutations of item and order information gains and losses. For each case, the first two letters indicate the information recalled on the previous trial $(k-1$, row value), whereas the second two letters indicate the information recalled on the current trial ( $k$, column value). 
Table 1 Item/Order Curves

\begin{tabular}{ccccc}
\hline & \multicolumn{4}{c}{ Trial $k$} \\
\cline { 2 - 5 } Trial $k-1$ & io & Io & iO & IO \\
\hline io & ioio & ioIo & - & ioIO \\
Io & Ioio & IoIo & - & IoIO \\
iO & - & - & - & - \\
IO & IOio & IOIo & - & IOIO \\
\hline
\end{tabular}

Note-All possible permutations of item and order information are shown. Cases along the main diagonal indicate no change from trial to trial and are, therefore, excluded from graphs. It is unclear how to measure $i O$ cases behaviorally, and they are therefore excluded from these analyses.

Correct item information is represented by an $I$, and incorrect item information is represented by an $i$. Similarly, correct relative order information is represented by an $O$, whereas incorrect relative order information is represented by an $o$. Not all of the 16 possible cases are informative. The cases involving order information without item information cannot be measured behaviorally with any standard methods. These cases have thus been excluded from all the analyses. The cases along the main diagonal represent no change in trial-to-trial behavior, so these have been excluded from the graphs, because they can be derived from the data showing gains and losses. This leaves six critical cases-three representing information gains and three representing information losses:

ioIO: gaining item and order information,

ioIo: gaining item information, incorrect order information,

IoIO: gaining order information, maintaining item information,

IOio: losing item and order information,

Ioio: losing item information, did not have order information,

IOIo: losing order information, maintaining item information.

One can illustrate the various cases described above by using example trials of recall. Given an example list ABSENCE, HOLLOW, PUPIL, DARLING, RIVER, RAILWAY, WINDOW, on Trial 1 the participant may recall ABSENCE, HOLLOW, WINDOW, RAILWAY. The items ABSENCE, HOLLOW, and WINDOW have all been gained in the correct relative order, so they are of the ioIO case. The item RAILWAY, however, is in the wrong relative order and is, therefore, of the ioIo case. On Trial 2, the participant may recall ABSENCE, PUPIL, HOLLOW, DARLING. The item HOLLOW is now in the wrong relative order and is now in the IOIo case. The item RAILWAY, which was in the wrong relative order on the previous trial, was not recalled on this trial. It is, therefore, of the Ioio case. On Trial 3, the participant might recall ABSENCE, HOLLOW, PUPIL, RIBBON, WINDOW. Now HOLLOW is back in the correct relative order and is of the IoIO case. The item DARLING, however, was in the correct relative order on the previous trial and has been forgotten on the current trial. It is therefore of the IOio case.

Plotting these six cases provides detailed information on the gains and losses of item and order information on each trial. Figure 2A shows the item/order analysis for serial recall of consonants. Participants generally gain item and order information together (ioIO: dotted line, top subpanel). This curve decreases over learning, since there are fewer items that can be gained. If an item is gained without order information, either it can be lost on a subsequent trial, or the order information can be gained on a subsequent trial. Given that there is so little forgetting (see bottom subpanel), it is evident that the latter is generally true-gaining item information and gaining order information trade off over trials, with more item gains (iolo: solid line, top subpanel) early in learning and more order gains (IoIO: dashed line, top subpanel) later in learning. Thus, the linear learning curve is formed predominantly from the ioIO curve, with the ioIo and $I O I O$ curves adding a small amount later in learning to supplement the decrease in the $i o I O$ curve. Figure $2 \mathrm{~B}$ reveals a similar pattern for the acquisition of 19-word lists.

The nature of the serial recall task places some constraints on the form of the item/order functions. Participants start out with no knowledge of the items on the list and must end with both item and order information for all of the list items. This means that the three curves plotting information losses must start and end at zero. The degree to which these points are not zero is a product of the Vincentization. The IoIO curve (gaining order information, maintaining item information) must start at zero, because one does not start off with any items in the wrong order. Furthermore, the iolo curve (gaining item information, incorrect order information) must end at zero; else, the criterion of having item and order information for all the items would not have been reached.

One could conditionalize the item/order analysis on the basis of the number of possible transitions for each case given what was recalled on the previous trial. We performed this analysis on the data presented in Figure 2B and found that the rates of forgetting item information and forgetting order information remain constant across trials (Ioio, $p=.61 ;$ IOIo, $p=.48)$, as do the rates of gaining item information and order information (ioIo, $p=$ $.07 ; \mathrm{IoIO}, p=.76$; the first and last trials are excluded because these points are undefined or must be equal to one). Although such conditional analyses can be informative, they can also be misleading. For example, if one conditionalizes the analysis of each transition, it would appear that item gains increase over trials because the number of available items is decreasing. However, participants actually gain roughly the same number of items on a given trial, regardless of list length. This is readily seen in looking at the unconditionalized analysis in Figures $2 \mathrm{~A}$ and $2 \mathrm{~B}$.

Using the unconditionalized item/order analysis of gains and losses across trials, we can now evaluate the predictions made by the associative chaining and posi- 

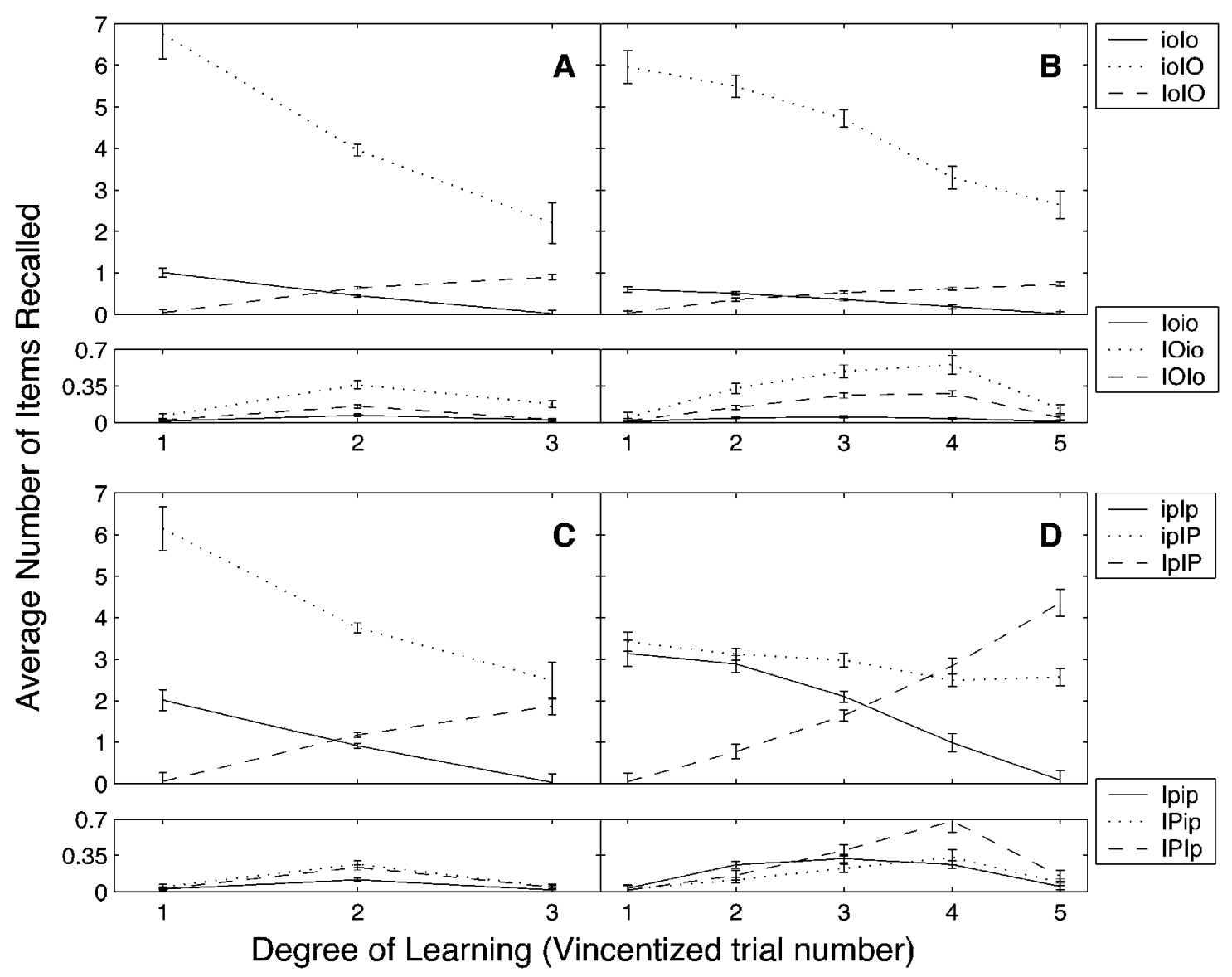

Figure 2. Item/order curves. (A) Serial recall of consonants, relative order scoring. (B) Serial recall of words, relative order scoring. (C) Serial recall of consonants, positional scoring. (D) Serial recall of words, positional scoring. Error bars represent $95 \%$ confidence intervals calculated according to the methods of Loftus and Masson (1994). The trials of learning are Vincentized (see the text for an explanation).

tional coding models discussed in the introduction. The chaining model predicts that all items will be learned with item and order information gained together, with or without forgetting depending on the implementation (Lewandowsky \& Murdock, 1989). This model cannot account for the items learned with item and order information gained on separate trials. The positional model would allow some items to be learned in the incorrect order in early trials, with order information being gained on later trials (Lee \& Estes, 1981). Although both models predict certain aspects of the data, the positional model is the most qualitatively accurate.

In the preceding analysis, we examined gains and losses of item and relative order information. We can apply the same analysis by using a strict positional scoring method. In this case, we are analyzing the gains and losses of item and positional information. Figures $2 \mathrm{C}$ and 2D show the results of the item/positional analysis. Figure $2 \mathrm{C}$ shows the item/positional analysis for serial lists of consonants. This pattern of results is highly similar to that seen in the item/order analysis in Figure 2A.
Figure 2D, however, shows that the item/positional analysis of serial lists of words reveals a different picture. In early trials, an equal number of items are gained with item and positional information together and with item information alone. There is also a noticeable increase in the losing of item and positional information separately, although the overall amount of information lost is still rather small.

Whereas the results of the relative order scoring for recall of words are highly consistent with the results of the first study (using relative order or positional information), the positional scoring shows a very different pattern of results. This difference appears only subtly in the learning curves, but it is readily apparent in the comparison of the item/order analysis with the item/position analysis. This difference is most likely due to the use of oral recall in the second study, rather than typed recall, which makes later serial positions much more prone to error. For instance, it is more difficult in oral recall than in written recall to correctly recall an item in its correct serial position after several omissions of items at earlier 
serial positions. Thus, relative order scoring seems to be less sensitive than positional scoring to methodological issues that may produce differences in results for trivial reasons. Hence, relative order scoring is a robust method that allows comparisons among studies with widely varying methodologies. The remaining analyses in this article, therefore, will focus only on relative order scoring.

From the item/order analysis, we see that item information and order information are almost always learned together. We also see a paucity of information losses; it may be that serial organization protects items from forgetting. Such organization is not a feature of free recall, because participants recall items in different orders, until late in learning when a subjective organization emerges (Tulving, 1962). Waugh (1961) demonstrated that whereas free recall exhibits rapid gains of information early in learning, there is relatively little improvement later in learning. Serial recall, however, exhibits a more constant rate of learning that surpasses free recall in later trials.
Although it is not entirely clear whether order information is always actually gained or whether it is merely inferred at recall from other recalled items, it is clear that once an item is recalled in the correct order, that order information is retained on subsequent trials. If items are recalled in the incorrect order, they are placed in the correct order on a subsequent trial. It is as if later trials are used to fit the previously unlearned item and order information into the list of known information.

In Figure 3, we expand the relative order scoring from the item/order analysis shown in Figures $2 \mathrm{~A}$ and $2 \mathrm{~B}$ to look at various levels of performance. For both studies, we partitioned the data into a $2 \times 2$ matrix of across-subjects comparisons by within-subjects comparisons. We divided the participants into two categories based on the average number of trials it took them to learn the lists. Participants whose mean number of trials to criterion was less than or equal to the grand mean were labeled goodlearners, whereas those whose average was greater
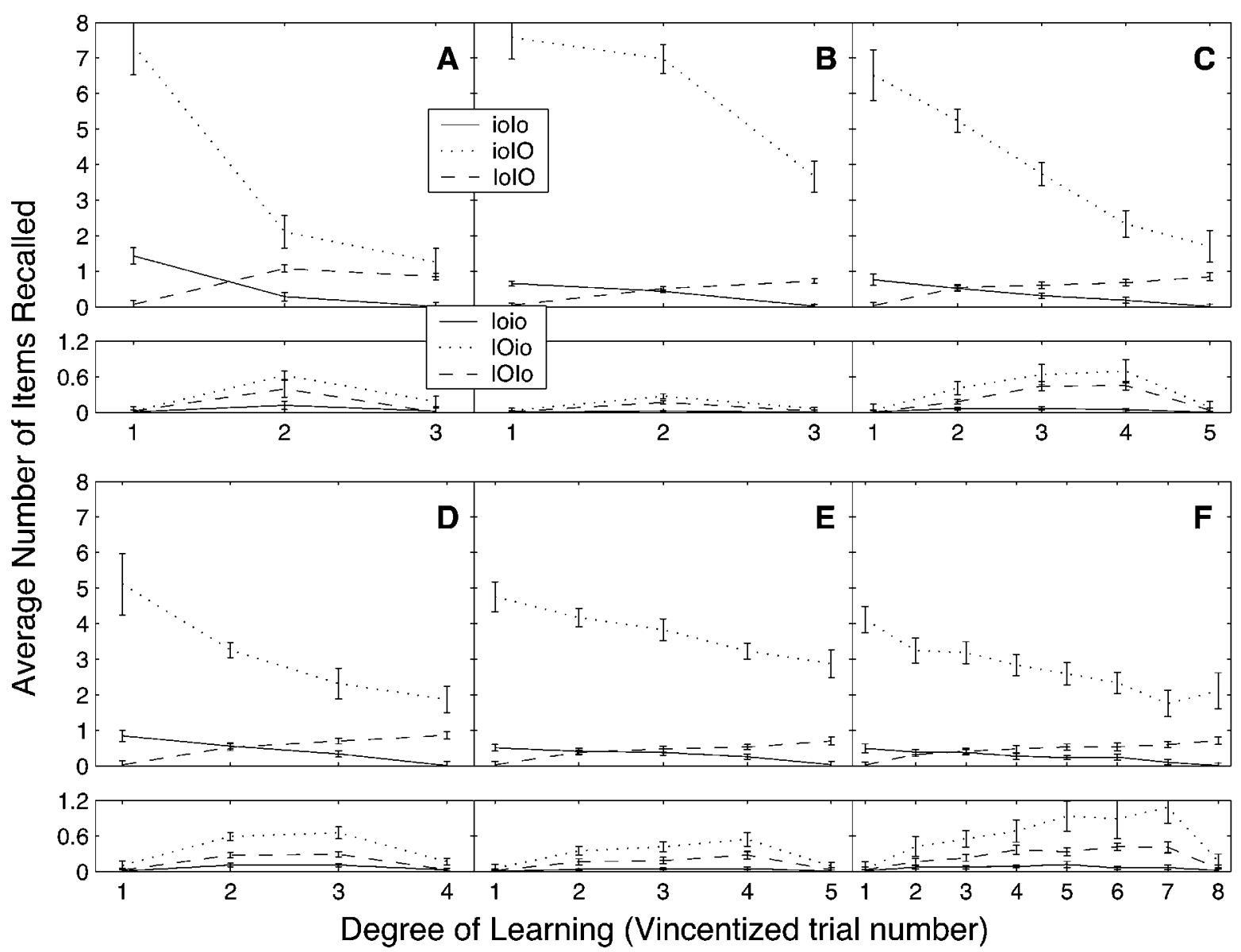

Figure 3. Item/order curves. (A) Serial recall of consonants (hard lists), good learners. (B) Serial recall of words (easy lists), good learners. (C) Serial recall of words (hard lists), good learners. (D) Serial recall of consonants (hard lists), poor learners. (E) Serial recall of words (easy lists), poor learners. (F) Serial recall of words (hard lists), poor learners. Error bars represent 95\% confidence intervals calculated according to the methods of Loftus and Masson (1994). The trials of learning are Vincentized (see the text for an explanation). 
than the grand mean were labeled poor learners. We then partitioned the lists for each participant into two categories, also based on the number of trials it took to learn the list: easy lists (learned in fewer trials than the participant's average) and hard lists (learned in more trials than the participant's average). The easy lists from the first study were excluded because they were completed in one trial and, therefore, are uninteresting in terms of learning curves or item/order analyses.

The six panels in Figure 3 show the item/order results for good and poor learners (rows) and for hard consonant lists and easy and hard word lists (columns). Looking across the data in Figure 3, it is only the speed of information acquisition that varies with performance. More precisely, poor learners forget more than good learners, but they still forget very little. Rather, they learn less new information per trial but still predominantly gain item and order information together. The same statements can be made when comparing the within-subjects data. Harder lists show slightly more forgetting, although forgetting is still very low, and less new information is gained per trial.

\section{Discussion}

The traditional analysis of serial recall - the learning curve-shows the rate of overall acquisition of the list items but collapses across the dynamics of individual item gains and losses. In this article, we extended Tulving's (1964) free recall analysis to serial recall, examining the trial-by-trial gains and losses of item and order information.

We carried out our item/order analysis on data sets from two serial recall experiments (Kahana \& Caplan, 2002; Kahana \& Jacobs, 2000). These two experiments used different stimuli, list lengths, and modalities. Despite these methodological differences, we see very similar results. Item information and order information were almost always learned together. In addition, information was not forgotten once it had been recalled. Only in a very few cases was item information learned alone, with order information learned on a subsequent trial. Given the extremely low rates of forgetting, these two curvesgaining item information alone and gaining order information for previously recalled items - trade off over trials, so that gains of item information were higher in early trials and gains of order information were higher in later trials. A further decomposition of this analysis for both within-subjects and across-subjects variations in performance shows the same pattern.

This analysis suggests that a common learning process underlies very different paradigms of symbolic sequence learning. Perhaps the sequential structure of the lists provides a mechanism that protects information from forgetting. When an item is recalled, it is maintained, and its relative order in the list is maintained with the addition of more recalled items. Items recalled without correct order information can, therefore, be placed in the correct order as more of the list is learned.

\section{REFERENCES}

Brown, G. D. A., Preece, T., \& Hulme, C. (2000). Oscillator-based memory for serial order. Psychological Review, 107, 127-181.

Burgess, N., \& Hitch, G. J. (1999). Memory for serial order: A network model of the phonological loop and its timing. Psychological Review, 106, 551-581.

Cowan, N., Saults, J. S., Elliott, E. M., \& Moreno, M. V. (2002). Deconfounding serial recall. Journal of Memory \& Language, 46, 153-177.

DREWNOWSKI, A., \& MURDock, B. B., JR. (1980). The role of auditory features in memory span for words. Journal of Experimental Psychology: Human Learning \& Memory, 6, 319-332.

Ebinghaus, H. (1913). Memory: A contribution to experimental psychology. New York: Teachers College, Columbia University. (Original work published 1885)

FARRELl, S., \& LEWANDOWSKy, S. (2002). An endogenous distributed model of ordering in serial recall. Psychonomic Bulletin \& Review, 9, 59-79.

HARCUM, E. R. (1975). Serial learning and paralearning: Control processes in serial acquisition. New York: Wiley.

Henson, R. N. A. (1998). Short-term memory for serial order: The start-end model. Cognitive Psychology, 36, 73-137.

Kahana, M. J., \& CaPlan, J. B. (2002). Associative asymmetry in probed recall of serial lists. Memory \& Cognition, 30, 841-849.

KAHANA, M. J., \& JACOBS, J. (2000). Interresponse times in serial recall: Effects of intraserial repetition. Journal of Experimental Psychology: Learning, Memory, \& Cognition, 26, 1188-1197.

Knoedler, A. J., Hellwig, K. A., \& Neath, I. (1999). The shift from recency to primacy with increasing delay. Journal of Experimental Psychology: Learning, Memory, \& Cognition, 25, 474-487.

KRUEGER, W. C. F. (1929). The effect of overlearning on retention. Journal of Experimental Psychology, 12, 71-78.

LEE, C. L., \& ESTES, W. K. (1981). Item and order information in shortterm memory: Evidence for multilevel perturbation processes. Journal of Experimental Psychology: Human Learning \& Memory, 7, 149-169.

LEWANDOWsky, S., \& Murdock, B. B. (1989). Memory for serial order. Psychological Review, 96, 25-57.

LI, X., SCHWEICKERT, R., \& GANDOUR, J. (2000). The phonological similarity effect in immediate recall: Positions of shared phonemes. Memory \& Cognition, 28, 1116-1125.

LofTUS, G. R., \& MASSON, M. E. J. (1994). Using confidence intervals in within-subjects designs. Psychonomic Bulletin \& Review, 1, 476-490.

TuLVING, E. (1962). Subjective organization in free recall of "unrelated" words. Psychological Review, 69, 344-354.

TulviNG, E. (1964). Intratrial and intertrial retention: Notes toward a theory of free recall verbal learning. Psychological Review, 71, 219-237.

VINCENT, S. B. (1912). The function of the vibrissae in the behavior of the white rat. Behavior Monographs, 1(No. 5).

WARD, L. B. (1937). Reminiscence and rote learning. Psychological Monographs, 49, 64.

WAUGH, N. C. (1961). Free versus serial recall. Journal of Experimental Psychology, 62, 496-502.

WaUGH, N. C. (1963). Two methods for testing serial memorization. Journal of Experimental Psychology, 65, 215-216.

WORKMAN, W. G., \& ADAMS, O. S. (1950). Suggestions for simplifying the computation of Vincent curves. Journal of General Psychology, 43, 147-151.

\section{NOTES}

1. The mean number of trials to criterion rounded to the nearest integer was equal to the median number of trials to criterion.

2 . The same pattern of increasing linearity is observed when looking at the raw, un-Vincentized data.

(Manuscript received June 13, 2002; revision accepted for publication December 27, 2002.) 